\title{
Prediction of Perinatal Morbidity in IUGR Pregnancies using Doppler Blood Flow Studies
}

\author{
D. Naveen ${ }^{1}$, K. Karthikeyan ${ }^{2}$ \\ ${ }^{1}$ Assistant Professor, Department of Radiodiagnosis, ${ }^{2}$ Assistant Professor, Department of Radiodiagnosis, Dhanlakshmi \\ Srinivasan Medical College and Hospital, Siruvachur, Perambalur-621113, India
}

Corresponding author: Dr K. Karthikeyan, Assistant Professor, Department of Radiodiagnosis, Dhanlakshmi Srinivasan Medical College and Hospital, Siruvachur, Perambalur-621113, India

DOI: http://dx.doi.org/10.21276/ijcmsr.2019.4.3.18

How to cite this article: D. Naveen, K. Karthikeyan. Prediction of perinatal morbidity in IUGR pregnancies using doppler blood flow studies. International Journal of Contemporary Medicine Surgery and Radiology. 2019;4(3):C82-C85.

\section{A B S T R A C T}

Introduction: The colour flow Doppler imaging has already found its place in studying the pathological vascular flow patterns in intra-uterine growth restriction and high-risk pregnancy. Study aimed to record the Doppler indices of Fetal Umbilical and Middle Cerebral arteries in IUGR pregnancies, to correlate the Doppler findings with the perinatal outcome and tso determine and compare the diagnostic predictive value of Umbilical and Middle Cerebral artery Doppler and their ratio for the prediction of adverse perinatal outcome.

Material and methods: The present study was conducted in 90 high risk pregnancies in the Department of Radio-Diagnosis of Pondicherry Institute Of Medical Sciences and Hospital from August 2012 to August 2014 . Initial assessment consisted of a detailed history of the patient as per the proforma. The cases for the study were selected as per inclusion and exclusion criteria.Colour Doppler examination was done. Doppler indices and perinatal outcome were recorded.The Sensitivity, Specificity, Positive Predictive Value and Negative predictive value were determined for the above Doppler indices for prediction of any adverse perinatal outcome.

Results: Umbilical artery was found to be the most specific (79\%) and had the-highest positive predictive value. The middle cerebral artery was found to be the most sensitive (79\%) and had the highest negative predictive value (60\%).

Conclusion:The abnormality in blood flow indices directly correlate with the neonatal outcome in the form of low birth weight, low apgar score and high neonatal morbidity and mortality especially in cases of abnormal umbilical artery waveforms such as in reversed end diastolic flow or absent end diastolic flow in which early intervention is advised.

Keywords: Prediction of Perinatal Morbidity, IUGR Pregnancies, Doppler Blood Flow

\section{INTRODUCTION}

Intrauterine growth restriction (IUGR) is described as sonographic estimated fetal weight of less than 10th percentile for gestational age. ${ }^{1}$ IUGR appears to be "one the most common and complex problems in modern obstetrics." according to the American College of Obstetricians and Gynecologists ${ }^{2}$

Challenges met against in the current clinical management of IUGR include accurate diagnosis of the truly growthrestricted fetus followed by selection of appropriate fetal surveillance, and there by optimizing the timing of delivery. ${ }^{3-5}$ Doppler velocimetry of the umbilical artery helps in assessing the resistance to blood perfusion of the fetoplacental unit .As early as 14 weeks, it is found that low impedance in the umbilical artery permits continuous forward flow throughout the cardiac cycle. ${ }^{6}$

Maternal disorders or placental conditions that are known to obliterate small muscular arteries in the placental tertiary stem villi result in a progressive decrease in enddiastolic flow in the umbilical artery Doppler Waveform which later becomes absent and then reversed flow during diastole eventually. ${ }^{7}$ Reversed end-diastolic flow in the umbilical arterial circulation is an indication of dvanced stage of placental compromise and has been associated with obliteration of about $70 \%$ of arteries in placental tertiary villi. ${ }^{8,9}$ Absent flow or reversed end-diastolic flow in the umbilical artery are found commonly associated with severe IUGR and oligohydramnios. ${ }^{10,11}$

During an episode of fetal hypoxemia, central redistribution of blood flow results in increased blood flow to the brain, heart, and adrenal glands, and a reduction in flow to the peripheral circulations. This blood flow redistribution, known as the brain-sparing reflex, is always characterized by increased end-diastolic flow velocity which is reflected by a low Pulasatality index [PI] in the middle cerebral artery. ${ }^{12,14}$ Doppler assessment of brain sparing can also be assessed using the cerebroplacental ratio, defined as middle cerebral artery PI/umbilical artery PI.A fetus is considered to have fetal brain sparing when this ratio is $<5$ th percentile for gestational age. ${ }^{15,16}$

Even a slight suspicion of fetal growth restriction should 
lead to an adequate surveillance of fetal health and Doppler velocimetry plays a major role in that. Doppler study of the fetal umbilical and middle cerebral arteries helps us to predict the perinatal outcome and modify the management accordingly.

The following study was done to review the use of the Doppler ultrasound of the fetal Umbilical and Middle Cerebral arteries and its efficacy in predicting the perinatal outcome in high risk pregnancies.

\section{MATERIAL AND METHODS}

The present study was conducted in 90 high risk pregnancies in the Department of Radio-Diagnosis of Pondicherry Institute Of Medical Sciences and Hospital from August 2012 to August 2014 . Initial assessment consisted of a detailed history of the patient as per the proforma. The study population was selected on the basis of following inclusion and exclusion criteria.

Inclusion Criteria: Singleton pregnancy, Fetal gestational age of 31 wks or greater confirmed by prior ultrasound examinations., Pregnancy with one or more high risk factors which may cause fetal compromise, or pregnancy with clinical diagnosis of intra uterine growth restriction(of unknown cause) which may not have any other high risk factor.

The high risk pregnancies studied included patients with PIH, PIH with IUGR, IUGR of unknown cause, chronic hypertension, eclampsia, renal disease, gestational diabetes with PIH, bad obstetric history, preterm premature rupture of membranes, post dated pregnancy, Rh negative pregnancy and other medical disorders complicating pregnancy.

\section{Exclusion Criteria}

- Pregnancy with documented congenital abnormality or any documented chromosomal anomaly were excluded from the study.

- Pregnancies with multiple gestations were excluded from the study

- Patients whose perinatal outcome could not be followed as they delivered outside were also excluded from the study.

The cases for the study were selected as per criteria stated above. Prior confirmation of the gestational age was done with a basic Ultrasonogram . Thereafter the colour Doppler examination was done.

\section{Doppler Technique}

A single stage colour Doppler study was done with an Philips envisor and Siemens S2000 colour Doppler machines with the $3.5 \mathrm{MHz}$ sector probe. Each subject was examined in the semi -recumbent position after a $15 \mathrm{~min}$ rest in the left lateral position. The transducer was then placed on the mother's abdomen and the free floating umbilical cord was identified. The Doppler sample volume was placed in the mid portion of the umbilical cord Similarly a transverse view of the fetal brain was obtained at the level of the biparietal diameter. The transducer was then moved towards the base of the skull at the level of the lesser wing of sphenoid bone were the middle cerebral artery could be visualized as a major lateral branch of the circle of Willis. The pulsed Doppler sample was then placed on the middle portion of this vessel and the flow velocity waveforms were obtained (fig-1,2). The pulsatality index for both umbilical and middle cerebral artery along with any reduced, absent, reversed or increased diastolic flow (if any) in the umbilical or middle cerebral artery was noted and the results tabulated.

\section{Outcome Measured Doppler Indices 1) Umbilical Artery Pulsatility Index}

It was considered abnormal if the value was above the 95 th percentile for that gestational age according to the published indices data.

\section{2) Middle Cerebral Artery Pulsatality Index}

The pulsatality index was considered as abnormal if the value was below the 5 th percentile for that gestational age according to the published indices data.

\section{3) Middle cerebral artery / Umbilical artery pulsatality index ratio.lt was considered abnormal if MCA PI / UA PI was less than 1.08}

The pregnancies were followed and the final perinatal outcome of each was assessed by various intrapartum and neonatal indicators like Caesarian section for fetal distress, Apgar score $<7$ at $5 \mathrm{~min}$, stay in NICU > 8 days, still birth or perinatal death.

Perinatal outcome was considered as adverse by presence of one or more of these indicators

The Sensitivity, Specificity, Positive Predictive Value and Negative predictive value were determined for the above Doppler indices for prediction of any adverse perinatal outcome using SPSS software.

\section{RESULTS}

Of the 90 pregnancies 56 of them had complications and adverse perinatal outcome .

Total 39 patients had UA PI > 95th percentile. Out of these patients 32 patients (True positive) had an adverse perinatal outcome where as 7 patients (false positive) had a good outcome. Total of 51 patients had a normal UA PI. Among these 24 patients (false negative) had an adverse perinatal outcome whereas 27 patients (true negative) had a good outcome .

Total of 60 patients had MCA PI < 5th percentile. Out of these patients 44 (True positive) developed adverse perinatal outcome and 16 patients (false positive) had a good perinatal outcome. . A total of 30 patients had a normal MCA PI. Among these, 12 patients (false negative) had an adverse outcome whereas 18 patients (true negative) had a good perinatal outcome.

Out of total of 90 patients, 35 patients had abdnormal cerebroplacental ratio. Among them 26 patients (True positive) had adverse perinatal outcome and 9 patients (false positive) had a good perinatal outcome. A total of 55 patients had a normal cerebroplacental ratio. Among them 30 patients (false negative) had an adverse outcome whereas 25 patients (true negative) had a good perinatal outcome.

By using the results of Doppler study for analysis the test characteristics of PI values for UA, MCA and MCA/UA for prediction of any adverse outcome was determined as shown in table 1. The MCA Pulsatality index was the most sensitive (79\%). It was significantly more sensitive than UA PI and 
also that of MCA / UA PI Ratio. Middle cerebral artery also had the highest Negative Predictive Value (60\%). Umbilical artery had the highest specificity of $79 \%$ and was significantly more specific than MCA PI or that of MCA/UA PI Ratio. The umbilical artery also had the highest Postive Predictive Value (82\%).

\section{DISCUSSION}

The colour flow Doppler imaging has already found its place in studying the pathological vascular flow patterns in intrauterine growth restriction and high-risk pregnancy.Its indices provides important information on the hemodynamics of the vascular area under study. ${ }^{17}$ Circulatory changes which are detected reflects certain fetal Doppler waveforms, that predicts adverse perinatal outcome. ${ }^{18}$ However its exact role as a predictor of pregnancy induced hypertension, intrauterine growth restriction, the resulting preterm delivery and associated complications is still undergoing trials.

Even a slight suspicion of fetal growth restriction should lead to an adequate surveillance of fetal health and Doppler velocimetry plays a major role in that. Doppler study of the fetal umbilical and middle cerebral arteries helps us to predict the perinatal outcome and modify the management accordingly.

The following study has been done to review the use of the Doppler ultrasound of the fetal Umbilical and Middle Cerebral arteries and its efficacy in predicting the perinatal outcome in high risk pregnancies.

The MCA Pulsatality index was the most sensitive (79\%). It was significantly more sensitive than UA PI and also that of MCA / UA PI Ratio. Middle cerebral artery also had the highest Negative Predictive Value (60\%). Umbilical artery had the highest specificity of $79 \%$ and was significantly more specific than MCA PI or that of MCA/UA PI Ratio. The umbilical artery also had the highest Postive Predictive Value (82\%).

Several observational studies have explored cerebral redistribution (abnormal middle cerebral artery Doppler results and / or abnormal MCA/UA Doppler ratio) for prediction of perinatal outcome in high risk pregnancy as depicted in table 2. However it is difficult to compare the findings of these studies since definition of adverse perinatal outcome varied. Diverse end points were used.

Arduini and Rizzo ${ }^{18}$ studied the test characteristics of PI from UA, MCA and Renal Artery to predict adverse perinatal outcome in 120 small for gestation age fetuses. In $46.7 \%$ (56 of 120) of fetuses, there was at least one of the following adverse outcomes: Perinatal death, Caesarean for Fetal distress, 5min Apgar $<7$, asphyxia that necessitated admission to NICU for $>48 \mathrm{hrs}$. They found that MCA/ UA PI ratio was the best test when compared with MCA or UA PI respectively.However this difference could be due to the fact that Arduini et al took only SGA fetuses from the start of the study where as in my study I took all high risk pregnancies which were at the risk of developing Utero $\neg$ placental insufficiency irrespective of the fact whether IUGR was present or absent at the time of Doppler.

Similarly Dandolo Gramellini et $\mathrm{al}^{19}$ who studied the cerebro-umbilical ratio as a predictor of adverse perinatal outcome gave the sensitivity, specificity, positive predictive value and negative predictive value of P.1 of MCA, UA and cerebro-umbilical ratio .

our study shows that umbilical artery $\mathrm{Pl}$ is more sensitive than cerebroumbilical ratio in predicting adverse perinatal outcome in contrast to Gramellini et al.

In the study by K.W. Fong et $\mathrm{al}^{20}$ they concluded that a normal MCA P.I I was found to be helpful to identify the fetuses without a major adverse perinatal outcome, hence once the UA P.I I abnormal, it is suggested to better perform the MCA P.I to know the extent of brain sparing, there by stressing the importance of studying two vessels in the Doppler.

Lahkar et $\mathrm{al}^{21}$ study had depicted the positive predictive value of a test shows its accuracy and this in our study was higher for MCA Pl, which was $47 \%$. The sensitivity of the combined Doppler parameters was highest at $91.6 \%$ in predicting perinatal outcome.

Rozeta et $\mathrm{al}^{22}$ also showed that abnormal MCA/UA PI Doppler ratio is strongly correlated with worse fetal prognosis. Khanduri et $\mathrm{a}^{23}$ studied 60 high risk pregnancies and and compared the accuracy of Doppler indices with perinatal outcome. The umbilical artery PI had maximum sensitivity and specificity which had an diagnostic accuracy of $75 \%$ compared to the MCA PI and had an overall diagnostic accuracy of $52.8 \%$.

Marilola et $\mathrm{al}^{24}$ study, the comparison of selected Doppler indices revealed that $\mathrm{C} / \mathrm{U}$ showed the highest sensitivity in prediction of both the intrapartum abnormal FHR (74.1\%) and the adverse neonatal outcome (87.8\%).

Chitrarasan et a ${ }^{25}$ There was also a significant rise in perinatal morbidity with increasing grades of Doppler (P value 0.001). Absent end diastolic flow (EDF)/reversal in umbilical artery had high positive predictive value in predicting adverse fetal outcome.

The normal Doppler result has more importance than an abnormal Doppler result. The explanation for these observations is probably that fetal growth retardation can be either due to low intrinsic growth potential or due to defective placental nutritive and circulatory functions, of which Doppler can investigate only the circulatory components.

\section{CONCLUSION}

The present study noted an adverse fetal outcome in cases of high risk pregnancy which showed abnormal Doppler indices in the umbilical and middle cerebral arteries.

In suspected IUGR, the Umbilical artery PI will provide most useful information for differentiating fetus already compromised or likely to be compromised from those that are uncompromised. Because of low specificity, single abnormal MCA Doppler results is not accurate for timing delivery. Therefore in fetuses suspected to have IUGR, while an abnormal Umbilical Artery PI is a better predictor of adverse perinatal outcome than abnormal Middle Cerebral Artery PI and $\mathrm{C} / \mathrm{U}$ ratio. Thus the present study concludes that the abnormality in blood flow indices directly correlate with the neonatal outcome in the form of low birth weight, low apgar score and high neonatal morbidity and mortality especially in cases of abnormal umbilical artery waveforms 
such as in reversed end diastolic flow or absent end diastolic flow in which early intervention is advised.

\section{REFERENCES}

1. Battaglia FC, Lubchenko LO. A practical classification of newborn infants by weight and gestational age. J Pediatr 1967;71(1):159-63.

2. American College of Obstetricians and Gynecologists. Intrauterine growth restriction; ACOG practice bulletin no. 12. Washington, DC:ACOG; 2000.

3. Baschat AA. Arterial and venous Doppler in the diagnosis and management of early onset fetal growth restriction. Early Hum Dev 2005; 81 (3):877-87.

4. Baschat AA, Gembruch U, Reiss I, Gortner L, Weiner CP, Harman CR. Relationship between arterial and venous Doppler and perinatal outcome in fetal growth restriction. Ultrasound Obstet Gynecol 2000;16 (5):407-13.

5. Bilardo CM, Wolf H, Stigter RH, et al. Relationship between monitoring parameters and perinatal outcome in severe, early intrauterine growth restriction. Ultrasound Obstet Gynecol 2004;23 (2):119-25.

6. Fisk NM, MacLachlan N, Ellis C, Tannirandorn Y, Tonge HM, Rodeck CH. Absent enddiastolic flow in first trimester umbilical artery. Lancet 1988;2 (1):12567.

7. Trudinger BJ, Stevens D, Connelly A, et al. Umbilical artery flow velocity waveforms and placental resistance: the effect of embolizations of the umbilical circulation. Am J Obstet Gynecol 1987;157 (2):1443-8.

8. Kingdom JC, Burrell SJ, Kaufmann P. Pathology and clinical implications of abnormal umbilical artery Doppler waveforms. Ultrasound Obstet Gynecol 1997;9 (4):271-86.

9. Morrow RJ, Adamson SL, Bull SB, Ritchie JW. Effect of placental embolization on the umbilical artery velocity waveform in fetal sheep. Am J Obstet Gynecol 1989;161 (6):1055-60.

10. McIntire DD, Bloom SL, Casey BM, Leveno KJ. Birth weight in relation to morbidity and mortality among newborn infants. N Engl J Med 1999;340 (3):1234-8.

11. Copel JA, Reed KL. Doppler ultrasound in obstetrics and gynecology, 1st ed. New York, NY: Raven Press; 1995:187-98.

12. Mari G, Deter RL. Middle cerebral artery flow velocity waveforms in normal and smallfor- gestational age fetuses. Am J Obstet Gynecol 1992;166 (1):1262-70. Level II-3.

13. Kurmanavicius J, Florio I, Wisser J, et al. Reference resistance indices of the umbilical, fetal middle cerebral and uterine arteries at 24-42 weeks of gestation. Ultrasound Obstet Gynecol 1997;10 (3):112-20. Level II-2.

14. Behrman RE, Lees MH, Peterson EN, De Lannoy CW, Seeds AE. Distribution of the circulation in the normal and asphyxiated fetal primate.AmJ Obstet Gynecol 1970;108 (5):956-69.

15. Bahado-Singh RO, Kovanci E, Jeffres A, et al. The Doppler cerebroplacental ratio and perinatal outcome in intrauterine growth restriction. Am J Obstet Gynecol 1999;180 (7):750-6. Level II-3.
16. Cruz-Martinez R, Figueras F, Hernandez- Andrade E, Oros D, Gratacos E. Fetal brain Doppler to predict cesarean delivery for nonreassuring fetal status in term small-for-gestational- age fetuses. Obstet Gynecol 2011;117 (1): 618-26.

17. Kurjak A, Kupesic S, Zudenigo D. Doppler ultrasound in all three trimesters of pregnancy. Curr Opin Obstet Gynecol 1994; 6 (5):472-8.

18. Arduini D, Rizzo G. Doppler studies of deteriorating growth retarded fetuses. Curr Opin Obstet Gynecol 1993, 5 (3):195-2-3

19. Gramellini D, Folli MC, Raboni S, Vadora E, Merialdi A. Cerebral - Umbilical Doppler Ratio As a Predictor of Adverse Perinatal Outcome - Obstetrics and Gynecology 1992; 79 (1):416-20.

20. Fong KW, Ohlsson A, Hanah Me, Kingdom J et al Prediction of Perinatal Outcome in Fetuses Suspected to Have Intrauterine Growth Restriction. Doppler US Study of Fetal Cerebral, Renal, and Umbilial Arteries Radiology 1999;213 (2):681-689.

21. Lakhkar BN, Rajagopal KV, Gourisankar PT. Doppler prediction of adverse perinatal outcome in PIH and IUGR. Indian J Radiol Imaging. 2006;16(1):10-6.

22. Rozeta Shahinaj, Nikita Manoku, Enriketa Kroi Ilir TashaThe value of the middle cerebral to umbilical artery Doppler ratio in the prediction of neonatal outcome in patient with preeclampsia and gestational hypertension. Journal of Prenatal Medicine 2010; 4 (2): 17-21.

23. Khanduri Sachin, Parashari Umesh C., Bashir Shazia, Bhadury Samarjit, Bansal Anurag.Comparison of Diagnostic Efficacy of Umbilical Artery and Middle Cerebral Artery Waveform with Color Doppler Study for Detection of Intrauterine Growth RestrictionThe Journal of Obstetrics and Gynecology of India (JulyAugust 2013;63(4):249-255.

24. Mariola Ropacka-Lesiak, Tomasz Korbelak, Joanna Świder-Musielak, Grzegorz Breborowicz Cerebroplacental ratio in prediction of adverse perinatal outcome and fetal heart rate disturbances in uncomplicated pregnancy at 40 weeks and beyond Arch Med Sci 2015; 11(1): 142-148.

25. Chirtrarasan P, Kanagarameswarakumaran S. Role of obstetric doppler in prediction of adverse perinatal outcome in intrauterine growth retardation. Int $\mathrm{J} \mathrm{Adv}$ Med 2017;4 (6):529-33.

\section{Source of Support: Nil; Conflict of Interest: None}

Submitted: 23-06-2019; Accepted: 02-08-2019; Published online: 14-08-2019 